\title{
Interactive impacts of overconfidence and fairness concern on supply chain performance
}

\author{
Zhang, Z.J. ${ }^{a}$, Wang, P. ${ }^{\text {b. }}{ }^{\text {, }}$, Wan, M.Y. ${ }^{c}$, Guo, J.H. ${ }^{a}$, Luo, C.L. ${ }^{c}$ \\ ${ }^{a}$ School of Transportation and Logistics, East China Jiaotong University, Nanchang, P.R. China \\ ${ }^{b}$ Dongwu Business School, Soochow University, Suzhou, P.R. China \\ ${ }^{c}$ School of Information Management, Jiangxi University of Finance and Economics, Nanchang, P.R. China
}

\section{A B S T R A C T}

For exploring the interactive impacts of overconfidence and fairness concern on optimal decisions of manufacturer and retailer, we establish Stackelberg models with these two behavioural preferences in a two-echelon supply chain, wherein retailer has two behavioural preferences. The optimal equilibrium results are compared in different scenarios, namely the retailer with no behavioural preference, with single-behavioural preference and with the two behavioural preferences. Although previous literatures have proven that overconfidence or fairness concern has a negative influence on retailer, we find that the retailer always benefits from these two behavioural preferences, whether it is retail price, sales effort or utility. This is because when the overconfident degree is within a reasonable range, overconfidence and fairness concern have a positive influence on retailer's decision-making, and when the overconfident degree is high, the fairness concern preference can suppress the adverse effects caused by overconfidence. Compared with the preference of fairness concern, the overconfident preference plays a leading role in supply chain performance, which mainly manifests in retailer's decisions, utility and manufacturer profit. Moreover, the wholesale price and profit of the rational manufacturer may become worsen due to the fairness concern of retailer.
\end{abstract}

\section{ARTICLE INFO}

Keywords:

Supply chain;

Supply chain management;

Modelling;

Performance;

Overconfidence;

Fairness concern;

Behavioural operation;

Stackelberg game

*Corresponding author: wangpeng_wl@126.com (Wang, P.)

Article history:

Received 10 September 2020

Revised 8 October 2020

Accepted 12 October 2020

\section{References}

[1] Zhang, Z., Wang, P., Wan, M., Guo, J., Liu, J. (2020). Supply chain decisions and coordination under the combined effect of overconfidence and fairness concern, Complexity, Vol. 2020, Article ID 3056305, doi: 10.1155/2020/ 3056305.

[2] Lu, C.L. (2016). P\&G's collaborative supply chain transformation, Enterprise Management, Vol. 10, 79-80.

[3] Xiao, Q., Chen, L., Xie, M., Wang, C. (2020). Optimal contract design in sustainable supply chain: Interactive impacts of fairness concern and overconfidence, Journal of the Operational Research Society, 1-20, doi: 10.1080/ 01605682. 2020.1727784.

[4] Yan, X., Chong, H.-Y., Zhou, J., Sheng, Z., Xu, F. (2020). Fairness preference based decision-making model for concession period in PPP projects, Journal of Industrial \& Management Optimization, Vol. 16, No. 1, 11-23, doi: 10.3934/jimo.2018137.

[5] Li, M. (2019). Overconfident distribution channels, Production and Operations Management, Vol. 28, No. 6, 1347 1365, doi: $10.1111 /$ poms.12981.

[6] Nie, T., Du, S. (2017). Dual-fairness supply chain with quantity discount contracts, European Journal of Operational Research, Vol. 258, No. 2, 491-500, doi: 10.1016/j.ejor.2016.08.051.

[7] Russo, J.E., Schoemaker, P.J.H. (1992). Managing overconfidence, Sloan Management Review, Vol. 33, No. 2, 7-17.

[8] Ren, Y., Croson, D.C, Croson, R.T.A. (2017). The overconfident newsvendor, Journal of the Operational Research Society, Vol. 68, No. 5, 496-506, doi: 10.1057/s41274-016-0103-5. 
[9] Kirshner, S.N., Shao, L. (2019). The overconfident and optimistic price-setting newsvendor, European Journal of Operational Research, Vol. 277, No. 1, 166-173, doi: 10.1016/i.ejor.2019.02.023.

[10] Doyle, J., Ojiako, U., Marshall, A., Dawson, I., Brito, M. (2020). The anchoring heuristic and overconfidence bias among frontline employees in supply chain organizations, Production Planning \& Control, 1-18, doi: 10.1080/ 09537287.2020 .1744042$.

[11] Liu, B., Cai, G., Tsay, A.A. (2014). Advertising in asymmetric competing supply chains, Production and Operations Management, Vol. 23, No. 11, 1845-1858, doi: 10.1111/poms.12090.

[12] Liu, W., Shen, X., Wang, D. (2018). The impacts of dual overconfidence behavior and demand updating on the decisions of port service supply chain: A real case study from China, Annals of Operations Research, Vol. 291, 565604, doi: 10.1007/s10479-018-3095-5.

[13] Xu, L., Shi, X., Du, P., Govindan, K., Zhang, Z. (2019). Optimization on pricing and overconfidence problem in a duopolistic supply chain, Computers \& Operations Research, Vol. 101, 162-172, doi: 10.1016/i.cor.2018.04.003.

[14] Kahneman, D., Knetsch, J.K., Thaler, R. (1986). Fairness as a constraint on profit seeking: Entitlements in the market, The American Economic Review, Vol. 76, No. 4, 728-741.

[15] Cui, T.H., Raju, J.S., Zhang, Z.J. (2007). Fairness and channel coordination, Management Science, Vol. 53, No. 8, 1303-1314, doi: $10.1287 /$ mnsc.1060.0697.

[16] Caliskan-Demirag, O., Chen, Y., Li, J. (2010). Channel coordination under fairness concerns and nonlinear demand, European Journal of Operational Research, Vol. 207, No. 3, 1321-1326, doi: 10.1016/j.ejor.2010.07.017.

[17] Li, Q.-H., Li, B. (2016). Dual-channel supply chain equilibrium problems regarding retail services and fairness concerns, Applied Mathematical Modelling, Vol. 40, No. 15-16, 7349-7367, doi: 10.1016/i.apm.2016.03.010.

[18] Wang, Y., Yu, Z., Shen, L. (2019). Study on the decision-making and coordination of an e-commerce supply chain with manufacturer fairness concerns, International Journal of Production Research, Vol. 57, No. 9, 2788-2808, doi: $10.1080 / 00207543.2018 .1500043$.

[19] Pan, K., Cui, Z., Xing, A., Lu, Q. (2020). Impact of fairness concern on retailer-dominated supply chain, Computers \& Industrial Engineering, Vol. 139, Article No. 106209, doi: 10.1016/i.cie.2019.106209.

[20] Karray, S. (2013). Periodicity of pricing and marketing efforts in a distribution channel, European Journal of Operational Research, Vol. 228, No. 3, 635-647, doi: 10.1016/j.ejor.2013.02.012.

[21] Zeng, L., Wang, J., Hu, Y. (2018). Retailer channel decisions of consumer electronics supply chain in a competitive environment, Tehnički Vjesnik - Technical Gazette, Vol. 25, No. 6, 1819-1828, doi: 10.17559/TV-20181101140 915.

[22] Mukhopadhyay, S.K., Yao, D.-Q., Yue, X. (2008). Information sharing of value-adding retailer in a mixed channel hi-tech supply chain, Journal of Business Research, Vol. 61, No. 9, 950-958, doi: 10.1016/i.jbusres.2006.10.027.

[23] Zheng, Z.L., Bao, X. (2019). The investment strategy and capacity portfolio optimization in the supply chain with spillover effect based on artificial fish swarm algorithm, Advances in Production Engineering \& Management, Vol. 14, No. 2, 239-250, doi: 10.14743/apem2019.2.325.

[24] Jian, M., Wang, Y.L. (2018). Decision-making strategies in supply chain management with a waste-averse and stockout-averse manufacturer, Advances in Production Engineering \& Management, Vol. 13, No. 3, 345-357, doi: 10.14743/apem2018.3.295.

[25] Chen, K.G., Song, X.F., Wang, X.Y., Huang, M. (2016). Joint pricing and production decisions with the overconfident sales agent, Journal of Systems \& Management, Vol. 25, No. 3, 468-476.

[26] Fehr, E., Schmidt, K.M. (1999). Theory of fairness, competition, and cooperation, The Quarterly Journal of Economics, Vol. 114, No. 3, 817-868, doi: 10.1162/003355399556151.

[27] Zheng, X.-X., Li, D.-F., Liu, Z., Jia, F., Sheu, J.-B. (2019). Coordinating a closed-loop supply chain with fairness concerns through variable-weighted Shapley values, Transportation Research Part E: Logistics and Transportation Review, Vol. 126, 227-253, doi: 10.1016/i.tre.2019.04.006.

[28] Ancarani, A., Di Mauro, C., D’Urso, D. (2016). Measuring overconfidence in inventory management decisions, Journal of Purchasing and Supply Management, Vol. 22, No. 3, 171-180, doi: 10.1016/i.pursup.2016.05.001. 


\title{
Interaktivni vplivi pretirane samozavesti in skrbi za pravičnost na uspešnost dobavne verige
}

\author{
Zhang, Z.J. ${ }^{a}$, Wang, P. ${ }^{\text {b. }}{ }^{*}$, Wan, M.Y. ${ }^{c}$, Guo, J.H. ${ }^{a}$, Luo, C.L. ${ }^{c}$ \\ aSchool of Transportation and Logistics, East China Jiaotong University, Nanchang, P.R. China \\ bDongwu Business School, Soochow University, Suzhou, P.R. China \\ 'School of Information Management, Jiangxi University of Finance and Economics, Nanchang, P.R. China
}

\section{POVZETEK}

Za raziskovanje interaktivnih vplivov pretirane samozavesti in skrbi za pravičnost na optimalne odločitve proizvajalca in trgovca vzpostavljamo Stackelbergove modele s tema dvema vedenjskima vzorcema v dvostopenjski dobavni verigi, pri čemer ima trgovec dva vedenjska vzorca. Rezultati optimalnega ravnotežja se primerjajo v različnih scenarijih, in sicer trgovec brez vedenjskih vzorcev, $\mathrm{z}$ enim vedenjskim vzorcem in $\mathrm{z}$ obema vedenjskima vzorcema. Čeprav so prejšnje raziskave pokazale, da pretirana samozavest ali skrb za pravičnost negativno vplivata na trgovca, ugotovimo, da ima trgovec od teh dveh vedenjskih vzorcev vedno koristi, ne glede na to, ali gre za maloprodajno ceno, prodajni napor ali uporabnost. To je zato, ker kadar je stopnja pretirane samozavesti v razumnem obsegu, pretirana samozavest in skrb za pravičnost pozitivno vplivata na odločanje trgovca, kadar pa je stopnja pretirane samozavesti visoka, lahko skrb za pravičnost zatre škodljive učinke, ki jih povzroči pretirana samozavest. V primerjavi z vedenjskim vzorcem skrbi za pravičnost ima vzorec pretirane samozavesti vodilno vlogo pri uspešnosti dobavne verige, kar se kaže predvsem v odločitvah trgovca, koristnosti in dobičku proizvajalca. Poleg tega se lahko veletrgovska cena in dobiček racionalnega proizvajalca znižata zaradi trgovčeve skrbi za pravičnost.
\end{abstract}

\section{PODATKI O ČLANKU}

Ključne besede:

Dobavna veriga;

Upravljanje dobavne verige;

Modeliranje;

Izvedba;

Pretirana samozavest;

Skrb za pravičnost;

Vedenjsko obnašanje;

Stackelbergova igra

*Kontaktna oseba: wangpeng_wl@126.com (Wang, P.)

Zgodovina članka:

Prejet 10. septembra 2020

Popravljen 8. oktobra 2020

Sprejet 12. oktobra 2020 